\title{
18. Werthaltungen und freiwilliges Engagement
}

\author{
Oliver Huxhold \& Doreen Müller
}

\section{Kernaussagen}

Menschen, denen der Wert Solidarität wichtig ist, engagieren sich anteilig häufiger. Auch wenn man Unterschiede hinsichtlich Bildung und finanziellen, sozialen und gesundheitlichen Ressourcen berücksichtigt, sind Personen, die es für wichtig halten, anderen Menschen zu helfen, häufiger freiwillig tätig als Menschen, denen Solidarität weniger wichtig ist.

Menschen, denen der Wert Kreativität wichtig ist, engagieren sich anteilig häufiger. Unabhängig von ihrer Bildung und ihren finanziellen, sozialen und gesundheitlichen Ressourcen engagieren sich Personen, die Wert darauf legen ihre Kreativität zu entwickeln, besonders häufig.

Menschen, denen der Wert Sicherheit wichtig ist, engagieren sich anteilig seltener. Personen, die Sicherheit für ein erstrebenswertes Gut halten, sind seltener freiwillig tätig als Menschen, die Sicherheit für nicht so wichtig halten. Der Unterschied zwischen diesen Gruppen bleibt auch dann bestehen, wenn man Unterschiede in der Bildung und in finanziellen, sozialen und gesundheitlichen Ressourcen berücksichtigt.

Bei Frauen hat die Einstellung zu Sicherheit einen stärkeren Einfluss auf das Engagement als bei Männern. Frauen, die Sicherheit als besonders wichtig erachten, engagieren sich anteilig deutlich seltener freiwillig als Frauen, die diesem Wert weniger Bedeutung beimessen. Bei Männern ist das Engagementverhalten weniger stark vom Wert Sicherheit beeinflusst.

Menschen unterschiedlichen Alters werden von verschiedenen Werthaltungen unterschiedlich stark in ihrem Engagement beeinflusst. Sicherheit weist insbesondere im mittleren Erwachsenenalter einen starken Zusammenhang mit dem freiwilligen Engagement auf. Die Werte Solidarität und Kreativität haben vor allem im höheren Erwachsenenalter eine große Bedeutung für die Ausübung freiwilliger Tätigkeiten.

\subsection{Einleitung}

Werte oder Werthaltungen werden in der Literatur als Prinzipien verstanden, an denen Menschen situationsübergreifend ihr Handeln ausrichten oder auch im Nachhinein rechtfertigen (Schwartz \& Bilsky 1987, 1990; Welzel 2009). Im Gegensatz zu Motiven, die häufig als Begründungen für eine konkrete Verhaltensweise angesehen werden, wirken Werte allgemeiner. Sie können sich in einer ganzen Reihe von mehr oder weniger konkreten Zielen und Verhaltensweisen zeigen. Ein weiterer Unterschied zwischen Werten und Motiven besteht darin, dass Werte immer eine normative Komponente enthalten. Mit Hilfe der persönlichen Werte können Ziele und Handlungen in die Kategorien gut und schlecht eingeordnet werden (Hofer, Reinders \& Fries 2010). Nach Schwartz und Bilsky (1987) entspringen unsere Werte universellen menschlichen Bedürfnissen, jedoch werden sie durch verschiedene Kulturen

(C) Der/die Autor(en) 2017

J. Simonson et al. (Hrsg.), Freiwilliges Engagement in

Deutschland, Empirische Studien zum bürgerschaftlichen

Engagement, DOI 10.1007/978-3-658-12644-5_19 
und Institutionen, wie zum Beispiel in der Familie oder der Schule, unterschiedlich vermittelt und geformt. In der Forschung wird angenommen, dass die Ausprägung von bestimmten Werten in Kindheit und Jugend entwickelt werden und dass diese Werthaltungen das Handeln über den ganzen Lebenslauf hinweg beeinflussen (Rokeach \& Ball-Rokeach 1989; Schwartz \& Bilsky 1987; Welzel 2009).

Diese Definition legt nahe, dass Werthaltungen auch die Aufnahme und Beibehaltung einer freiwilligen Tätigkeit mitbestimmen. Allerdings sind Werthaltungen nicht unabhängig voneinander. Zum Beispiel legen Menschen, denen Sicherheit wichtig ist, häufig Wert auf Tradition (Schwartz \& Bilsky 1990). In der deutschen Forschung zu Werten und Engagement wurden deshalb häufig die einzelnen Werthaltungen zu übergeordneten Wertetypen gruppiert (Klages \& Gensicke 2002). Dieser Ansatz hat den Vorteil, dass er die Beziehungen zwischen den einzelnen Werten berücksichtigt. Werte, die stark zusammenhängen, werden in einem Wertetyp zusammengefasst. Dadurch kann der gesamtgesellschaftliche Wandel in individuellen Werthaltungen gut charakterisiert werden (Klages \& Gensicke 2006). Die Nutzung von Wertetypen in der Forschung birgt aber auch den Nachteil, dass nicht mehr klar erkennbar ist, welchen Stellenwert die einzelnen Werte für das Engagement haben. Aus diesem Grund betrachten wir hier einzelne Werthaltungen, um ihren jeweiligen Beitrag für das Engagement abzuschätzen.

Die internationale Engagementforschung hat sich im Vergleich zur deutschen Engagementforschung fast gänzlich auf den Zusammenhang zwischen einzelnen solidarischen Werten und freiwilligem Engagement fokussiert. Solidarische Werthaltungen drücken aus, dass eine Person das Wohl der Mitmenschen besonders stark im Auge hat. Verschiedene Untersuchungen zeigen, dass insbesondere Personen mit hoher Ausprägung in diesen solidarischen Werthaltungen anteilig häufiger und zeitlich intensiver freiwillig tätig sind als Menschen, die einen größeren Fokus auf individuelle Ziele legen (Grönlund, Holmes,
Kang, Cnaan, Handy, Brudney, Haski-Leventhal, Hustinx, Kassam, Meijs, Pessi, Ranade, Smith, Yamauchi \& Zrinščak 2011).

Um ein umfangreiches Verständnis der Einflüsse von Werthaltungen auf freiwilliges Engagement $\mathrm{zu}$ erlangen, reicht es jedoch nicht aus, sich ausschließlich auf solidarische Werte zu konzentrieren. Es gibt nämlich plausible Gründe für die Annahme, dass eine solidarische Haltung nicht der einzige Wert ist, der die Entscheidung, sich freiwillig zu engagieren, beeinflussen kann. Aus der Motivforschung (siehe Kapitel 15) ist bekannt, dass Menschen aus den unterschiedlichsten Antrieben heraus einer freiwilligen Tätigkeit nachgehen. Diesen konkreten Motiven können die verschiedensten allgemeineren Werthaltungen zugrunde liegen. Andere Werte wiederum könnten dem Engagement sogar abträglich sein. Daher sollte bei der Betrachtung von Werten und Engagement ein breiterer Blickwinkel eingenommen und mehrere unterschiedliche Werte untersucht werden.

In diesem Kapitel betrachten wir, ob Menschen, die großen Wert darauf legen die eigene Kreativität $\mathrm{zu}$ entwickeln, auch eher bereit sind sich zu engagieren, weil sie offen für neue Erfahrungen sind. Dabei könnten freiwillige Tätigkeiten, in denen viele Möglichkeiten zur Mitgestaltung und Mitsprache gegeben sind, für Menschen, denen es wichtig ist ihre Kreativität $\mathrm{zu}$ entwickeln, besonders interessant sein. Im Gegensatz dazu könnten Personen, denen Sicherheit besonders wichtig ist, weniger geneigt sein ein Engagement aufzunehmen, weil sie sich dazu möglicherweise in neue ungewohnte Situationen begeben müssten, oder weil ihnen der Erfolg des Engagements ungewiss erscheint. Aus diesen Überlegungen heraus untersuchen wir, ob die beiden genannten Werte, die bisher noch wenig Beachtung in der Engagement-Literatur erfahren haben, die Wahrscheinlichkeit sich zu engagieren beeinflussen. Zusätzlich werden wir untersuchen, ob eine solidarische Werthaltung tatsächlich den stärksten Einfluss auf das Engagementverhalten zeigt. 
Ein weiterer Grund dafür, die Untersuchung der Zusammenhänge zwischen Werten und Engagement über die Solidarität hinaus zu erweitern, liegt in der Tatsache, dass Werthaltungen innerhalb verschiedener gesellschaftlicher Gruppen höchst unterschiedlich verteilt sind. Folgerichtig könnten Unterschiede in individuellen Werthaltungen möglicherweise Gruppenunterschiede im Engagement erklären. In diesem Zusammenhang ist besonders interessant, dass bisherige Studien eine höhere Wichtigkeit solidarischer Einstellungen bei älteren im Vergleich zu jüngeren Menschen sowie bei Frauen im Unterschied zu Männern zeigen (Robinson 2013; Sawyerr, Strauss \& Yan 2005; Schwartz \& Rubel 2005). Doch trotz der stärkeren Orientierung von Älteren und Frauen an solidarischen Werten weisen diese beiden Bevölkerungsgruppen keine höheren Engagementquoten auf als jüngere Erwachsene und Männer, sondern sind sogar weniger häufig freiwillig engagiert (Einolf 2011; Windsor, Anstey \& Rodgers 2008). Wenn die persönliche Wichtigkeit von Kreativität und Sicherheit ebenfalls die Entscheidung beeinflusst, sich zu engagieren, könnte dies zum Teil erklären, warum ältere Menschen sich weniger häufig freiwillig engagieren, obwohl sie häufiger als jüngere Personen solidarisch eingestellt sind. So haben Untersuchungen gezeigt, dass die Werthaltung Offenheit für Neues, die positiv mit der Werthaltung Kreativität und negativ mit dem Wert Sicherheit verknüpft ist, bei älteren Bevölkerungsgruppen weniger stark ausgeprägt ist als bei jüngeren Menschen (Robinson 2013).

Weiterhin ist $\mathrm{zu}$ erwarten, dass individuelle Werthaltungen gerade im höheren Erwachsenenalter für das Engagement wichtiger werden, weil die Engagementgelegenheiten mit dem Übergang in den Ruhestand abnehmen (Freund, Nikitin \& Ritter 2009). Nehmen die Anstöße aus dem beruflichen Umfeld ab, könnten die Werte in den Vordergrund rücken und zu Eigeninitiative bei der Aufnahme einer freiwilligen Tätigkeit führen. Eine ähnliche Beziehung könnte auch für Frauen gelten. Wie bereits an anderer Stelle in diesem Bericht festgestellt (siehe Kapitel 16), sind Frauen möglicherweise auch deswegen weniger häufig freiwillig engagiert, weil sie über weniger Ressourcen und Zugangsmöglichkeiten verfügen als Männer. Mit anderen Worten, es ist möglicherweise schwieriger für Frauen als für Männer, sich zu engagieren. Unter diesen Bedingungen könnten es gerade diejenigen Frauen sein, die über ein hohes Maß an engagementförderlichen und ein niedriges $M a ß$ an engagementhinderlichen Werthaltungen aufweisen, die sich über diese Schwierigkeiten hinwegsetzen. Aus diesen Argumenten geht hervor, dass man erwarten kann, dass Werthaltungen eine größere Bedeutung für Frauen und Ältere in Bezug auf das Engagement haben als für Männer und jüngere Menschen. Dies würde empirisch bedeuten, dass die statistischen Zusammenhänge zwischen Werten und Engagementquoten bei Frauen und älteren Personen stärker wären als in den jeweiligen Vergleichsgruppen.

Im vorliegenden Kapitel werden wir im Abschnitt Datengrundlage zunächst zeigen, inwieweit sich Frauen und Männer und Personen verschiedener Altersgruppen voneinander in den individuellen Werthaltungen bezüglich Solidarität, Kreativität und Sicherheit unterscheiden. Danach werden wir drei Forschungsfragen untersuchen:

1. Welche Zusammenhänge existieren zwischen den Werten Solidarität, Kreativität und Sicherheit und dem Engagement? Unterscheiden sich die Anteile Engagierter bei Menschen mit unterschiedlich hohen Ausprägungen in den Werthaltungen Solidarität, Kreativität und Sicherheit? Weisen die Werte Solidarität, Kreativität und Sicherheit in verschiedenen gesellschaftlichen Gruppen (nach Geschlecht und Alter) unterschiedliche Zusammenhänge mit Engagement auf?

2. Verstecken sich hinter den Zusammenhängen zwischen Werten und dem Engagement andere Einflüsse? Sind die Zusammenhänge von Solidarität, Kreativität und Sicherheit für freiwilliges Engagement auch dann unter- 
schiedlich für verschiedene gesellschaftliche Gruppen, wenn andere Aspekte der Lebenssituation von Menschen berücksichtigt werden (multivariate Betrachtung)?

3. Welchen Stellenwert hat jede einzelne Werthaltung für das Engagement? Weist Solida- rität einen stärkeren Zusammenhang mit dem Engagement auf als die Werte Sicherheit und Kreativität, auch wenn man alle Werte gleichzeitig betrachtet und zusätzlich andere Aspekte der Lebenssituation berücksichtigt (multivariate Betrachtung)?

\subsection{Datengrundlage}

Die in diesem Kapitel berichteten deskriptiven Befunde basieren auf gewichteten Analysen. Für die Gewichtung wurden in allen Erhebungsjahren dieselben Gewichtungsmerkmale berücksichtigt. Dabei handelt es sich um die Designgewichtung, die sich auf die Auswahlwahrscheinlichkeit einer Person bezieht (Haushaltsgröße, Zahl der Festnetznummern und, nur für das Jahr 2014, auch die Zahl der Mobilfunknummern) und Gewichtungsmerkmale, die Abweichungen der Stichprobenverteilung von der Grundgesamtheit in bestimmten Dimensionen ausgleichen (Bundesland, Gemeindegrößenklassen, Geschlecht, Altersgruppen). Von einer Hinzunahme weiterer Gewichtungsmerkmale wie etwa des Bildungsstandes haben wir abgesehen, um die Vergleichbarkeit mit den bisher veröffentlichten Berichten zu erhalten.

Alle Teilnehmerinnen und Teilnehmer des Freiwilligensurveys werden gefragt:

Wie wichtig sind Ihnen folgende Dinge:

- Die eigene Phantasie und Kreativität entwickeln (Werthaltung Kreativität)

- Nach Sicherheit streben (Werthaltung Sicherheit)

- Sozial Benachteiligten und gesellschaftlichen Randgruppen helfen (Werthaltung Solidarität)

Die Befragten bewerten, wie wichtig ihnen Kreativität, Sicherheit und Solidarität auf einer fünfstufigen Skala sind, die von, sehr wichtig' bis zu ,gar nicht wichtig' reicht. In unseren Abbildungen unterscheiden wir übersichtshalber nur zwischen Personen, die bezüglich eines entsprechenden Wertes angeben, er sei Ihnen, sehr wichtig' oder ,eher wichtig', und denen, die dies nicht taten (die also ,teils/teils', eher nicht wichtig' beziehungsweise, gar nicht wichtig angaben). Die erste Kategorie bezeichnet eine Gruppe von Menschen, denen ein bestimmter Wert wichtig ist.

Im Folgenden werden Geschlechter- und Altersunterschiede im Bereich der Werthaltungen in der Wohnbevölkerung berichtet. Nur vor dem Hintergrund dieser Erkenntnisse lässt sich der Einfluss von Werten auf das freiwillige Engagement überzeugend abschätzen. Wie in der Einleitung beschrieben, erwarteten wir, dass Frauen und ältere Menschen eher solidarisch eingestellt sind als Männer und jüngere Personen. Weiterhin vermuteten wir, dass ältere Personen weniger Wert auf Kreativität und mehr Wert auf Sicherheit legen als jüngere Menschen. Die Unterschiede in den Werthaltungen sind in Tabelle 18-1 dargestellt.

Der Anteil der Personen mit hoher Ausprägung in einer solidarischen Werthaltung ist bei Frauen um 8,5 Prozentpunkte höher als bei Männern. Es gibt keine ausgeprägten Geschlechterunterschiede bezüglich der Werthaltung Kreativität. Jedoch ist der Anteil der Frauen mit einer hohen Ausprägung beim Wert Sicherheit etwas höher als bei Männern (2,7 Prozentpunkte). 
Tabelle 18-1: Anteile der Personen mit hoher Ausprägung in Solidarität, Kreativität und Sicherheit 2014, gesamt sowie nach Geschlecht und nach Altersgruppe

\begin{tabular}{lccc}
\hline & Solidarität & Kreativität & Sicherheit \\
\hline Gesamt & 60,0 & 73,1 & 78,7 \\
\hline Frauen & 64,1 & 73,6 & 80,0 \\
\hline Männer & 55,6 & 72,6 & 77,3 \\
\hline 14 bis 29 Jahre & 57,1 & 73,7 & 78,1 \\
\hline 30 bis 49 Jahre & 57,3 & 72,3 & 78,7 \\
\hline 50 bis 64 Jahre & 62,0 & 75,0 & 77,7 \\
\hline 65 Jahre und älter & 63,9 & 71,8 & 80,3 \\
\hline
\end{tabular}

Quelle: FWS 2014, gewichtet, eigene Berechnungen (DZA). Basis: Alle Befragten ( $n=28.385-28.493)$.

In Bezug auf Altersunterschiede nehmen wir an, dass ältere Menschen auf der einen Seite solidarischer sind, auf der anderen Seite aber weniger Wert auf Kreativität legen und ihnen Sicherheit wichtig ist. Die beobachteten Werte entsprechen tendenziell unseren Erwartungen. Die jüngste Altersgruppe und die Gruppe von Personen im Alter von 30 bis 49 Jahren unterscheiden sich nicht in ihren Werthaltungen. Die beiden ältesten Altersgruppen weisen das höchste Maß an solidarischen Werten auf. Der Unterschied zwischen Menschen, die 50 Jahre und älter sind, und jüngeren Personen beträgt etwa fünf Prozentpunkte. Menschen ab 65 Jahren sind noch etwas häufiger solidarisch eingestellt als die zweitälteste Gruppe. Entgegen den Erwartungen sind die Altersunterschiede im Wert Kreativität unregelmäßig. Die Gruppe der ab 65-Jährigen misst jedoch dem Wert, die eigene Kreativität zu entwickeln, im Durchschnitt weniger Wichtigkeit bei als die Gruppe der 50- bis 64-Jährigen. Dieser
Unterschied weist darauf hin, dass diese Altersgruppe möglicherweise weniger geneigt ist, neue Erfahrungen zu suchen, als jüngere Menschen. Im Gegensatz dazu ist Sicherheit der ältesten Altersgruppe wichtiger als allen anderen Gruppen. Diese Beobachtung könnte ein Hinweis darauf sein, dass sich die älteste betrachtete Altersgruppe weniger gern in neue, ungewohnte Situationen begibt als jüngere Gruppen. Bei der Interpretation der vergleichsweise niedrigen Ausprägung der Werthaltung Kreativität beziehungsweise der relativ hohen Ausprägung der Werthaltung Sicherheit in der ältesten Altersgruppe sollte man jedoch bedenken, dass die Altersunterschiede in diesen beiden Werthaltungen insgesamt ziemlich klein sind. Der Unterschied zwischen der ältesten Gruppe und den anderen Befragten beträgt im Wert Kreativität im Durchschnitt etwa drei Prozentpunkte und im Wert Sicherheit im Durchschnitt nur etwa einen Prozentpunkt. 


\subsection{Zusammenhänge zwischen Werthaltungen und Engagement}

Solidarität: Um den Zusammenhang zwischen dem Grad der Ausprägung des Wertes Solidarität und freiwilligen Engagement zu überprüfen, vergleichen wir den Anteil von Engagierten in der Gruppe von Menschen, die angeben, anderen zu helfen sei ihnen, eher wichtig' oder, sehr wichtig', mit der Häufigkeit des Engagements von
Personen, denen dieser Wert nicht so wichtig ist. Unterschiede zwischen Menschen mit hoher und niedriger Ausprägung im Wert Solidarität innerhalb der Geschlechter und innerhalb einzelner Altersgruppen werden zusätzlich miteinander verglichen (Abbildung 18-1).

Abbildung 18-1: Anteile Engagierter differenziert nach hoher und niedrigerer Wichtigkeit des Wertes Solidarität 2014, gesamt, nach Alter und nach Geschlecht

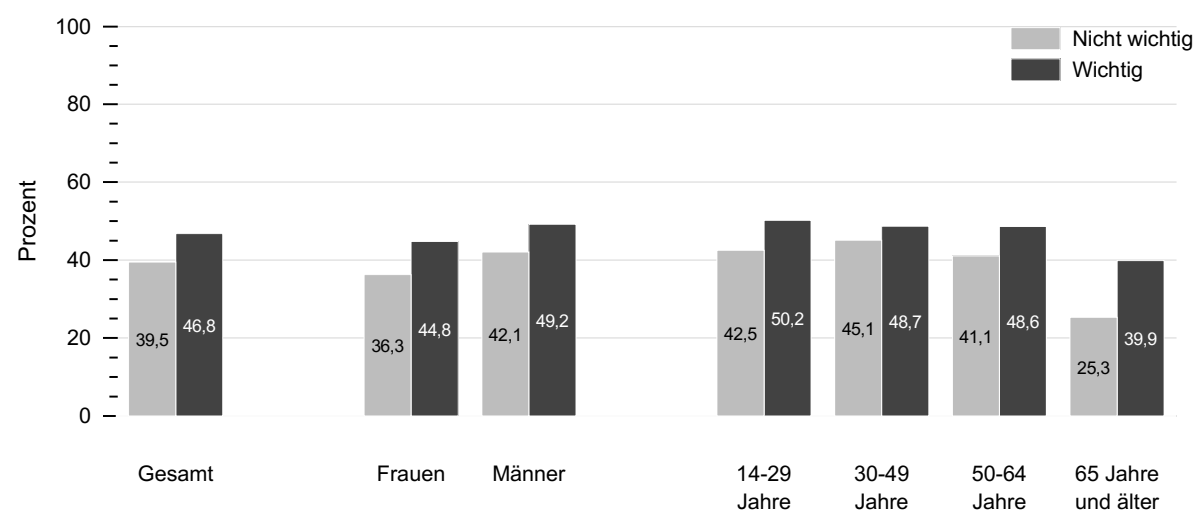

Quelle: FWS 2014, gewichtet, eigene Berechnungen (DZA). Basis: Alle Befragten ( $n=28.384-28.492$ ).

Insgesamt zeigt sich, dass sich Menschen, denen Solidarität wichtig ist, anteilig häufiger engagieren (46,8 Prozent) als Personen, die diesen Wert weniger wichtig finden (39,5 Prozent). Der Unterschied im Zusammenhang zwischen Solidarität und Engagement ist zwar bei Frauen leicht größer (8,5 Prozentpunkte) als bei Männern (7,1 Prozentpunkte), aber er unterscheidet sich nicht signifikant zwischen den Geschlechtern. Die Unterschiede zwischen Personen mit hoher beziehungsweise niedriger Ausprägung im Wert Solidarität sind jedoch signifikant unterschiedlich in den verschiedenen Altersgruppen. In der Gruppe der 30- bis 49-Jährigen ist der Unterschied am geringsten und beträgt nur 3,6 Prozentpunkte.
Bei Menschen, die 65 Jahre oder älter sind, ist er am größten und beträgt 14,6 Prozentpunkte.

Kreativität. Im Folgenden ist dargestellt, ob Menschen, denen es wichtig ist, die eigene Phantasie und Kreativität zu entwickeln, häufiger engagiert sind, als Personen, denen Kreativität nicht so wichtig ist. Wie in der Einleitung angesprochen, erwarteten wir, dass der Zusammenhang zwischen Kreativität und Engagement bei Frauen stärker ist als bei Männern und dass er über die Altersgruppen hinweg zunimmt. Aus diesen Gründen vergleichen wir die Unterschiede im Engagement zwischen Menschen mit hoher beziehungsweise geringerer Ausprägung des Wertes Kreativität zusätzlich zwischen den Geschlechtern und Altersgruppen (Abbildung 18-2). 
Abbildung 18-2: Anteile Engagierter differenziert nach hoher und niedrigerer Wichtigkeit des Wertes Kreativität 2014, gesamt, nach Alter und nach Geschlecht

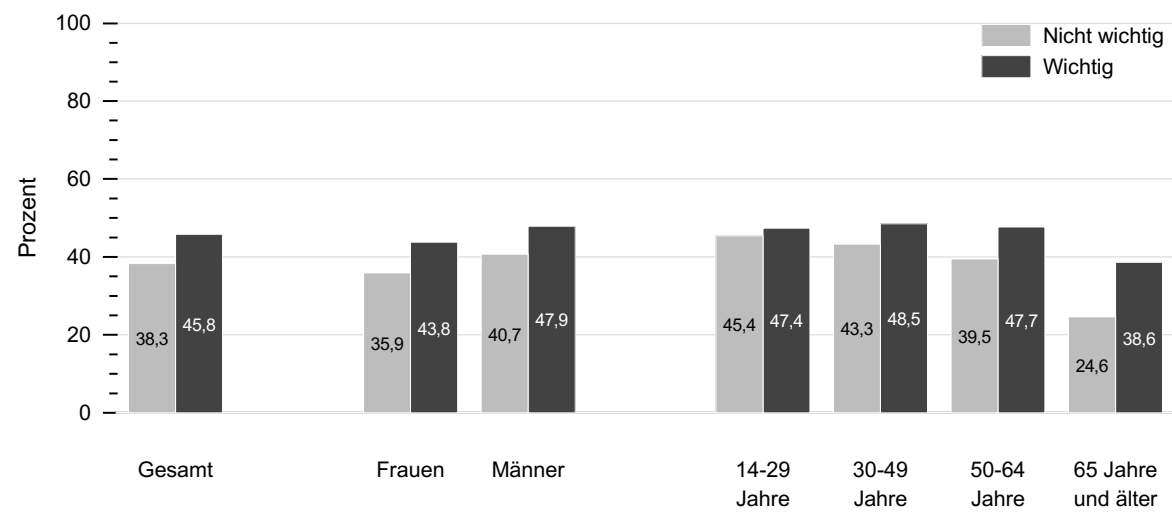

Quelle: FWS 2014, gewichtet, eigene Berechnungen (DZA). Basis: Alle Befragten ( $n=28.384-28.492$ ).

\section{Abbildung 18-3: Anteile Engagierter differenziert nach hoher und niedrigerer Wichtigkeit des Wertes Sicherheit 2014, gesamt, nach Alter und nach Geschlecht}

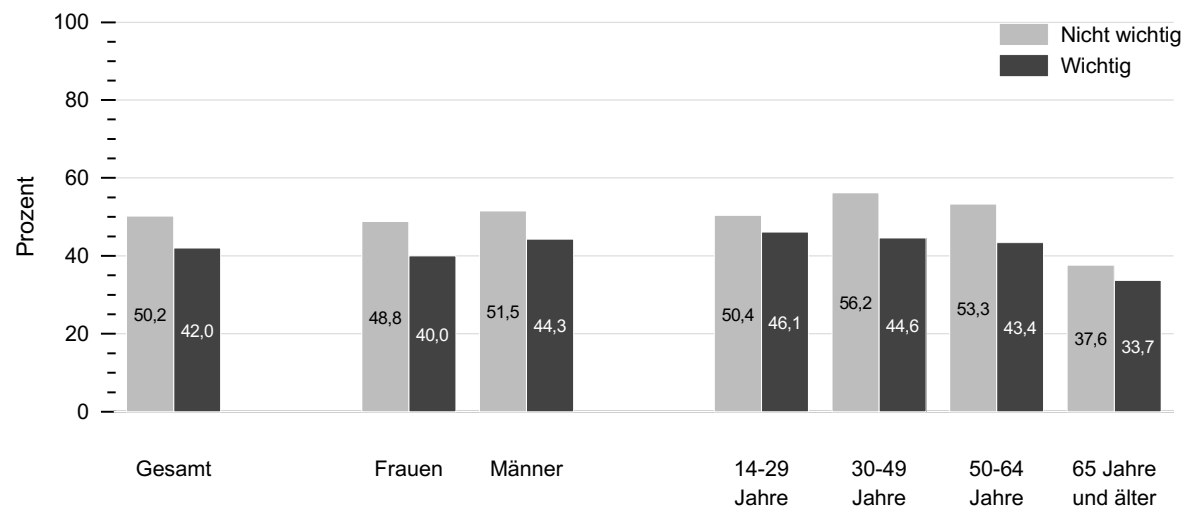

Quelle: FWS 2014, gewichtet, eigene Berechnungen (DZA). Basis: Alle Befragten ( $n=28.384-28.492$ ).

Menschen, denen Kreativität wichtig ist, engagieren sich häufiger (45,8 Prozent) als Personen, die darauf nicht so viel Wert legen (38,3 Prozent). Dieser Unterschied ist bei Männern und Frauen in etwa gleich stark ausgeprägt. Über die Altersgruppen hinweg nimmt der Unterschied in den Engagementquoten zwischen Menschen mit hoher beziehungsweise niedriger Ausprägung des Wertes Kreativität zu. In der jüngsten
Altersgruppe beträgt er 2,0 Prozentpunkte. In der ältesten Gruppe macht der Unterschied 14,0 Prozentpunkte aus.

Sicherheit. Als nächstes wird untersucht, ob sich Menschen, die großen Wert auf Sicherheit legen, anteilig weniger häufig engagieren, als Personen, die diesem Wert weniger Wichtigkeit beimessen. Ausgeprägtere Unterschiede der Engagementraten von Menschen mit hoher 
beziehungsweise niedriger Ausprägung dieser Werthaltung, differenziert nach Geschlecht und Alter, lassen auf einen stärkeren Zusammenhang zwischen Sicherheit und Engagement bei bestimmten Personengruppen schließen (Abbildung 18-3).

Die Analysen zeigen, dass je wichtiger den Personen der Wert Sicherheit ist, desto geringer ist die Wahrscheinlichkeit, sich zu engagieren.
Bei Frauen ist der Unterschied zwischen Menschen mit hoher beziehungsweise niedriger Ausprägung im Wert Sicherheit signifikant größer als bei Männern. Vergleicht man die Unterschiede in den Engagementquoten zwischen den Altersgruppen erkennt man, dass diese Unterschiede in der jüngsten und der ältesten Altersgruppe weniger stark ausfallen als in den beiden mittleren Altersgruppen.

\subsection{Verbergen sich hinter den Zusammenhängen zwischen Werten und dem Engagement andere Einflüsse?}

Die bisherigen Analysen deuten an, dass die $\mathrm{Zu}-$ sammenhänge der Werte Solidarität, Kreativität und Sicherheit mit dem Engagement bei Frauen und Männern und in verschiedenen Altersgruppen möglicherweise unterschiedlich stark sind. Allerdings sind diese Unterschiede bislang nicht multivariat (das heißt bei gleichzeitiger Berücksichtigung verschiedener Einflussgrößen) statistisch abgesichert worden. Zu diesem Zweck nutzen wir multivariate logistische Regressionen. Mit Hilfe dieser statistischen Methode lässt sich die Frage beantworten, ob eine abhängige Variable mit zwei Ausprägungen (in diesem Fall Engagement ja/nein) von mehreren unabhängigen Variablen gleichzeitig beeinflusst wird. In unseren Berechnungen berücksichtigen wir auch die Interaktionen zwischen den Wertevariablen und dem Geschlecht beziehungsweise der Altersgruppe. Eine signifikante Interaktion zeigt an, dass der Zusammenhang zwischen Werten und dem Engagement besonders ausgeprägt in einer Gruppe ist. Um sicher zu gehen, dass mögliche Zusammenhänge nicht auf Gruppenunterschieden in Ressourcen zurückzuführen sind, nehmen wir Bildung, subjektives Einkommen, subjektive Gesundheit und subjektive Netzwerkqualität als Kontrollvariablen in unsere Modelle auf. Zusätzlich wird für mögliche Stichprobenverzerrungen kontrolliert, indem die Variablen Bundesland und Festnetz- versus Mobilfunkstichprobe ebenfalls in den Rechnungen berücksichtigt werden.

In Tabelle 18-2 sind die Ergebnisse dreier logistischer Regressionen wiedergegeben. Für jede der drei Fokusvariablen (Solidarität, Kreativität und Sicherheit) wurde jeweils ein eigenes Modell gerechnet. Alle Fokusvariablen gehen als metrische Variablen in die Berechnung ein. Die abhängige Variable ist immer Engagement ( $\mathrm{ja} /$ nein). Die Wahrscheinlichkeit, sich zu engagieren, wird in jeder Regression mit jeweils einer der Fokusvariablen sowie Geschlecht, Alter und Bildung der Personen sowie den Interaktionen zwischen Geschlecht und Alter mit der Fokusvariablen vorhergesagt. Als Referenzgruppe für die Testung der Effekte wurde die Gruppe der Männer im Alter von 50 bis 64 Jahren mit mittlerem Bildungsniveau gewählt. Es wurde dabei diejenige Gruppe als Bezugspunkt gewählt, die im Alter und in der Bildung in einer mittleren Position liegt (daher wurde die Gruppe der Personen im mittleren Alter mit mittlerer Bildung gewählt). Zudem wurde die Gruppe der Männer als Referenzgröße gewählt, um die Ergebnisse für die Gruppe der Frauen explizit darstellen zu können (wird die Referenzgruppe Frauen gewählt, kann kein Koeffizient für Frauen ausgewiesen werden). Die Vergleichsgruppe für die Interaktionen ist die Gruppe der Männer im Alter von 50 bis 64 
Jahren mit mittlerem Bildungsniveau, die eine mittlere Ausprägung in den Fokus- und Kontrollvariablen haben. Berichtet werden unstandardisierte Koeffizienten. Ein negativer Koeffizient bedeutet, dass die Wahrscheinlichkeit sich $\mathrm{zu}$ engagieren, kleiner ist als in der Vergleichs- gruppe. Ein positiver Koeffizient heißt, dass die Engagementwahrscheinlichkeit größer ist. Berichtet werden nur signifikante Koeffizienten, nicht signifikante Koeffizienten werden mit drei Strichen dargestellt.

Tabelle 18-2: Ergebnisse logistischer Regressionen, Vorhersage freiwilligen Engagements durch drei Fokusvariablen (Solidarität, Kreativität, Sicherheit), Geschlecht, Alter, Bildung sowie Interaktionen der Fokusvariablen mit Geschlecht und Alter, 2014

\begin{tabular}{|c|c|c|c|}
\hline & Solidarität & Kreativität & Sicherheit \\
\hline Fokusvariable & $0,18 * * *$ & $0,10 * * *$ & $-0,22 * * *$ \\
\hline \multicolumn{4}{|l|}{ Geschlecht (Referenz: Männer) } \\
\hline Frauen & $-0,25 * * *$ & $-0,22 * * *$ & $-0,19 * * *$ \\
\hline \multicolumn{4}{|l|}{ Alter (Referenz: 50 bis 64 Jahre) } \\
\hline 14 bis 29 Jahre & $-0,13 * * *$ & $-0,14 * * *$ & $-0,12 * *$ \\
\hline 30 bis 49 Jahre & --- & --- & --- \\
\hline 65 Jahre und älter & $-0,26 * * *$ & $-0,23 * * *$ & $-0,22 * * *$ \\
\hline \multicolumn{4}{|l|}{ Bildung (Referenz: Mittlere Bildung) } \\
\hline Noch Schüler/in & $0,51 * * *$ & $0,52 * * *$ & $0,47^{* * *}$ \\
\hline Niedrige Bildung & $-0,39 * * *$ & $-0,39 * * *$ & $-0,40 * * *$ \\
\hline Hohe Bildung & $0,41 * * *$ & $0,40 * * *$ & $0,35 * * *$ \\
\hline \multicolumn{4}{|l|}{ Interaktion mit Geschlecht } \\
\hline Fokusvariable*Frauen & --- & --- & $-0,07^{* *}$ \\
\hline \multicolumn{4}{|l|}{ Interaktion mit Alter } \\
\hline Fokusvariable*14-29 Jahre & --- & $-0,09 *$ & $0,09 *$ \\
\hline Fokusvariable*30-49 Jahre & $-0,07^{*}$ & --- & --- \\
\hline Fokusvariable*65 Jahre und älter & $0,08^{*}$ & --- & $0,09 * *$ \\
\hline Pseudo $\mathrm{R}^{2}$ & $0,06 * * *$ & $0,06 * * *$ & $0,06 * * *$ \\
\hline Anzahl gültiger Fälle & $27.862 * * *$ & $27.956 * * *$ & $27.962 * * *$ \\
\hline
\end{tabular}

Quelle: FWS 2014, ungewichtet, eigene Berechnungen (DZA). Kontrolliert für subjektives Einkommen, Netzwerkqualität, subjektive Gesundheit sowie Design-Variablen (Bundesland, Kontakt über Festnetz/Mobilfunk). $* p<0,05, * * p<0,01, * * * p<0,001,---$ nicht signifikant.

Die multivariaten Analysen bestätigen weitestgehend die bisherigen Beobachtungen. Unabhängig von der Bildung neigen Männer und Frauen eher dazu sich zu engagieren, wenn sie solidarisch eingestellt sind und auf die Entwicklung der eigenen Kreativität Wert legen. Der Effekt von Solidarität und Kreativität auf das Engagement ist für bei- de Geschlechter gleich stark. Die Interaktionen von Geschlecht mit den beiden Fokusvariablen sind nicht signifikant. Im Gegensatz dazu gibt es einen Geschlechterunterschied im Einfluss des Wertes Sicherheit auf das Engagementverhalten (Koeffizient der Interaktion -0,07). Für Frauen ist die Wahrscheinlichkeit eines Engagements 
geringer, wenn sie eine hohe Ausprägung im Wert Sicherheit haben, als bei Männern, die genauso viel Wert auf Sicherheit legen.

Solidarität: Der Unterschied in den Engagementquoten zwischen Menschen mit hoher und solchen mit niedriger Ausprägung des Wertes Solidarität ist bei Menschen, die 50 Jahre und älter sind, signifikant höher als in der Gruppe der 30-49-Jährigen. Dies wird daran deutlich, dass die Interaktion der Fokusvariable Solidarität und der Altersgruppe 30 bis 49 Jahre statistisch signifikant ist (Koeffizient -0,07). Zusätzlich ist der Unterschied zwischen solidarisch und weniger solidarisch eingestellten Menschen in den Engagementquoten in der ältesten Gruppe signifikant ausgeprägter als in der zweitältesten (der Koeffizient für die Interaktion der Fokusvariablen Solidarität mit der Altersgruppe 65 Jahre und älter beträgt 0,08). Für Menschen, die 65 Jahre oder älter sind, hat die Werthaltung Solidarität also einen größeren Einfluss auf die Wahrscheinlichkeit sich zu engagieren.

Kreativität: Auch die persönliche Haltung zur Kreativität wird mit dem Alter wichtiger für das Engagement. Allerdings unterscheidet sich nur die Gruppe der 14- bis 29-Jährigen in der Stär- ke des Zusammenhanges zwischen Kreativität und Engagement signifikant von den anderen Altersgruppen (Interaktionskoeffizient -0,09). In dieser Gruppe scheint der Wert, den man der Entwicklung der eigenen Kreativität beimisst, keine größere Rolle für die Aufnahme oder die Aufrechterhaltung einer freiwilligen Tätigkeit zu spielen. Die stetige Zunahme des Zusammenhanges über die Altersgruppen, die in der bivariaten Analyse beobachtet wurde, scheint vornehmlich durch Bildungsunterschiede entstanden zu sein. Für Menschen mit hoher Bildung ist es wichtiger, die eigene Kreativität zu entwickeln, als für Menschen mit niedriger Bildung und der Anteil an hochgebildeten Personen ist bei den älteren Altersgruppen niedriger.

Sicherheit: Der Wert Sicherheit scheint insbesondere im mittleren Erwachsenenalter von großer Bedeutung für das Engagement zu sein. Die Stärke des negativen direkten Zusammenhanges zwischen Alter und Engagement wird durch die positiven Interaktionseffekte abgeschwächt. Das gilt sowohl für die jüngste Altersgruppe (Interaktionskoeffizient 0,09 ) als auch für die älteste (Interaktionskoeffizient 0,09), nicht aber für die beiden mittleren Altersgruppen.

\subsection{Welchen Stellenwert hat jede einzelne Werthaltung für das Engagement?}

Der Ausgangspunkt für die Fragestellungen in diesem Kapitel besteht in der Annahme, dass die individuelle Haltung zur Solidarität sicherlich mit freiwilligem Engagement verknüpft ist, aber dass auch andere Werthaltungen in diesem Zusammenhang berücksichtigt werden sollten. Die ersten bisherigen Analysen entsprechen diesen Erwartungen. Menschen, die eine hohe solidarische Werthaltung haben, engagieren sich häufiger als Personen mit niedriger Ausprägung in diesem Wert. Aber auch Menschen, die einen hohen Wert auf die Entwicklung ihrer Kreativität legen und solche, die nicht so sehr nach Sicherheit streben, sind häufiger freiwillig tätig als Menschen, denen Sicherheit wichtig und Kreativität eher unwichtig sind. Wie im vorangehenden Abschnitt gezeigt, findet man diese $\mathrm{Zu}$ sammenhänge auch, wenn man für Unterschiede bei der Bildung und finanziellen, sozialen und gesundheitlichen Ressourcen kontrolliert. Allerdings können wir in diesen Analysen nicht untersuchen, welchen Stellenwert jede einzelne Werthaltung im Zusammenspiel zwischen Werten und Engagement hat.

Wie in der Einleitung erwähnt, existieren individuelle Werthaltungen nicht unabhängig 
voneinander. Menschen, die solidarisch eingestellt sind, halten auch häufig Kreativität für wichtig (Klages \& Gensicke 2002). Um den unabhängigen Beitrag jeder einzelnen Werthaltung $\mathrm{zu}$ bestimmen, überprüfen wir die univariaten Zusammenhänge mit Hilfe einer multivariaten logistischen Regression, bei der alle drei Werte gleichzeitig als metrische Variablen in die Vorhersage des Engagements eingehen. Ein signifikanter Zusammenhang zwischen einem der Werte und dem Engagement in diesem Modell zeigt den individuellen Beitrag dieser Werthaltung bei der Vorhersage sich freiwillig zu engagieren an. Zusätzlich kontrollieren wir in dieser Analyse auch für Geschlecht, Altersgruppe, subjektive Gesundheit, subjektives Einkommen, Netzwerkqualität und Schulbildung. Damit können wir zufällige Zusammenhänge ausschließen, die auf Korrelationen dieser Variablen mit den Werthaltungen beruhen. In einem letzten Schritt untersuchen wir, ob sich die Stärke der Zusammenhänge zwischen den Werten und der Wahrscheinlichkeit, sich zu engagieren, unterscheidet. Dazu überprüfen wir mit Hilfe von Modelltests, ob die Höhe der Koeffizienten in der logistischen Regression, die die Werte Solidarität, Kreativität und Sicherheit mit der Engagementwahrscheinlichkeit verbindet, signifikant verschieden voneinander sind. Die Ergebnisse der Regression sind in Tabelle 18-3 dargestellt.

Tabelle 18-3: Ergebnisse einer logistischen Regression: Vorhersage freiwilligen Engagements durch die Werthaltungen Solidarität, Kreativität und Sicherheit und Geschlecht, Alter sowie Bildung, 2014

\begin{tabular}{lc}
\hline & Engagement \\
\hline Solidarität & $0,18^{* * *}$ \\
\hline Kreativität & $0,09 * * *$ \\
\hline Sicherheit & $-0,25^{* * *}$ \\
\hline Geschlecht (Referenz: Männer) & $-0,23^{* * *}$ \\
\hline Frauen & $-0,09 * *$ \\
\hline Alter (Referenz: 50 bis 64 Jahre) & $0,10^{* * *}$ \\
\hline 14 bis 29 Jahre & $-0,21^{* * *}$ \\
\hline 30 bis 49 Jahre & \\
\hline 65 Jahre und älter & $0,44^{* * *}$ \\
\hline Bildung (Referenz: Mittlere Bildung) & $-0,37^{* * *}$ \\
\hline Noch Schüler/in & $0,34^{* * *}$ \\
\hline Niedrige Bildung & $0,06^{* * *}$ \\
\hline Hohe Bildung & $27.635^{* * *}$ \\
\hline Pseudo R 2 & \\
\hline Anzahl gültiger Fälle & \\
\hline
\end{tabular}

Quelle: FWS 2014, ungewichtet, eigene Berechnungen (DZA). Kontrolliert für subjektives Einkommen, Netzwerkqualität, subjektive Gesundheit sowie Design-Variablen (Bundesland, Kontakt über Festnetz/Mobilfunk). $* \mathrm{p}<0,05, * * \mathrm{p}<0,01, * * * \mathrm{p}<0,001$.

Die abhängige Variable in diesem Model ist das Engagement (nein/ja). Als Vergleichsgruppe dienen Männern im Alter von 50 bis 64 Jahren, die über eine mittlere Bildung verfügen. Ein positi- ver Koeffizient zeigt eine erhöhte Wahrscheinlichkeit an sich zu engagieren.

Die Analyse, in der alle Werthaltungen gleichzeitig in die Berechnung eingehen, be- 
stätigt die zuvor berichteten Zusammenhänge. Auch nach Kontrolle von weiteren Aspekten stehen alle drei Werthaltungen unabhängig voneinander und signifikant mit der Wahrscheinlichkeit, sich zu engagieren, in Zusammenhang. Solidarität ist also nicht die einzige Werthaltung, die im Kontext des freiwilligen Engagements bedeutsam ist. Menschen, die es wichtig finden, anderen zu helfen, Menschen, die Wert auf die Entwicklung ihrer Kreativität legen und solche, denen Sicherheit weniger wichtig ist, üben eher eine freiwillige Tätigkeit aus als andere Personen.

\subsection{Fazit}

Die vorliegenden Analysen zeigen deutlich, dass Solidarität ein wichtiger Wert ist, der Menschen motivieren kann, eine freiwillige Tätigkeit auszuüben. Aber zu einem umfassenden Verständnis des Zusammenhanges zwischen Werten und dem Engagement gehört es, noch weitere individuelle Werthaltungen zu berücksichtigen. Das Bedürfnis, die eigene Kreativität zu entwickeln, kann für einige Personen unabhängig von ihrem Bedürfnis, anderen Menschen zu helfen, ein Ansporn sein eine freiwillige Tätigkeit aufzunehmen. Daher kann es eine Strategie der Engagementförderung sein, die Gestaltungsmöglichkeiten der Engagierten im Engagement stärker zu betonen. Auf die Wichtigkeit der Ausweitung dieser Spielräume hat bereits die Enquete-Kommission ,Zukunft des Bürgerschaftlichen Engagements' hingewiesen (Enquete-Kommission ,Zukunft des Bürgerschaftlichen Engagements 2002).

Weiterhin sind Menschen, die viel Wert auf Sicherheit legen, anteilig weniger häufig engagiert, als Personen, die Sicherheit einen weniger großen Wert beimessen, und zwar auch dann, wenn diese Menschen Solidarität für ein hohes Gut halten. Die vergleichenden Untersuchungen zeigen sogar, dass der hindernde Einfluss eines hohen Sicherheitsbedürfnisses stärker auf das
Unsere abschließenden Modelltests zeigen interessanterweise, dass Solidarität nicht der stärkste Einflussfaktor ist. Die Werthaltung Sicherheit zeigt einen signifikant stärkeren Zusammenhang mit dem Engagement (der Koeffizient ist -0,25) als die individuelle Wichtigkeit, solidarisch $\mathrm{zu}$ sein (der Koeffizient ist 0,18). Der Unterschied zwischen den beiden Koeffizienten ist statistisch signifikant, auch wenn man die unterschiedlichen Vorzeichen berücksichtigt. Kreativität (der Koeffizient ist 0,09) wiederum weist einen signifikant schwächeren Zusammenhang mit dem Engagement auf als Solidarität und Sicherheit.

Engagementverhalten wirkt als eine mögliche Motivation durch eine solidarische Haltung. Möglicherweise beginnen Menschen, denen $\mathrm{Si}$ cherheit besonders wichtig ist, weniger häufig ein freiwilliges Engagement, weil sie sich nicht gern in eine neue, für sie ungewohnte Umgebung begeben möchten und sie sich nicht sicher sind, ob sie sich den damit verbundenen Herausforderungen aussetzen wollen. Folgt man dieser Argumentation, bedeutet dies, dass es sinnvoll wäre, nicht nur an die Solidarität zu appellieren, wenn man versuchen möchte mehr Menschen für das freiwillige Engagement zu gewinnen. Vielmehr könnte es hilfreich sein, darzulegen, dass die Engagementsituation sich nicht so sehr vom gelebten Alltag unterscheidet. Möglicherweise spricht es Menschen mit hohem Sicherheitsempfinden an, wenn darauf hingewiesen wird, dass die Fähigkeiten und Kenntnisse, die sie im Beruf, in der Kindererziehung oder in der Organisation ihres Alltagslebens erworben haben, auch im Engagement gebraucht werden.

Eine solche Strategie, die Sicherheitsbedürfnisse berücksichtigt, könnte insbesondere bei der Einbindung von Frauen in das freiwillige Engagement erfolgreich sein. Unsere Analysen haben gezeigt, dass sie im Durchschnitt einen etwas größeren Wert auf Sicherheit legen als 
Männer. Zusätzlich wirkt sich ein hohes Sicherheitsbedürfnis bei Frauen noch negativer auf die Bereitschaft zur Ausübung eines Engagements aus als bei Männern.

Die gruppenspezifische Bezugnahme auf Werte könnte auch bei Anwerbungsstrategien für unterschiedliche Altersgruppen zum Zuge kommen. Bei älteren Menschen scheint dabei die Betonung der solidarischen Aspekte des Engagements besonders vielversprechend. Die Wertschätzung von Solidarität steigt über die Altersgruppen hinweg an und entfaltet bei Menschen im Alter ab 65 Jahren den stärksten Einfluss auf die Wahrscheinlichkeit, engagiert zu sein. Der Ruhestand kann gekennzeichnet sein von einer verringerten Angebotsstruktur für Aktivitäten im Allgemeinen und bei einigen Menschen in diesem Alter können sich schon gesundheitliche Einschränkungen bemerkbar machen. Deshalb sind in dieser Altersphase insbesondere solche Menschen freiwillig tätig, die hohe Ausprägungen in solidarischen Werten haben und aus sich heraus sehr motiviert sind.

Interessant ist, dass der Wert Sicherheit in der ältesten Altersgruppe keine besondere Rolle im Zusammenhang mit dem Engagement spielt. Vermutlich liegt es daran, dass im höheren Erwachsenenalter seltener neue freiwillige Tätigkeiten begonnen werden. Wenn ältere Menschen engagiert sind, üben sie in der Mehrzahl ihre freiwillige Tätigkeit schon relativ lange aus (siehe Kapitel 12). Allerdings haben unsere Untersuchungen auch gezeigt, dass Älteren der Wert Sicherheit etwas wichtiger ist. Dementsprechend könnte es hilfreich sein, auf Sicherheitsbedürfnisse einzugehen, wenn man bislang nicht-engagierte ältere Personen anregen möchte freiwillig tätig zu werden.

In der jüngsten Altersgruppe der 14- bis 29-Jährigen hängen individuelle Werthaltungen am wenigsten stark mit Engagement zusammen. Vielleicht wird die Entscheidung, sich zu engagieren in dieser Altersphase eher von Gelegenheiten, die über ihr soziales Netz an sie herangetragen werden, oder Motivlagen (zum Beispiel das Bedürfnis Teil einer Gruppe zu sein oder individuelle Interessen) bestimmt (siehe Kapitel 15).

Natürlich lässt sich in unseren querschnittlichen Analysen nicht ausschließen, dass nicht individuelle Werthaltungen die Wahrscheinlichkeiten ein Engagement aufzunehmen oder beizubehalten bestimmen, sondern dass für einen Teil der Befragten die Erfahrungen aus der freiwilligen Tätigkeit dazu führen, ihre Werthaltungen zu ändern (Hofer et al. 2010). Besonders für engagierte Menschen im Jugend- und jungen Erwachsenenalter ist der Einfluss des Engagements auf die Werthaltung möglich, weil ihre individuellen Werthaltungen möglicherweise noch nicht so gefestigt sind (Reinders \& Youniss 2006). Nichtsdestotrotz gehen bekannte Wertetheorien davon aus, dass individuelle Werthaltungen im Zuge der Sozialisation vermittelt werden und danach über den ganzen Lebenslauf relativ stabil bleiben (Rokeach \& Ball-Rokeach 1989; Schwartz \& Bilsky 1987; Welzel 2009). Deshalb besteht in den älteren Altersgruppen möglicherweise eine höhere Wahrscheinlichkeit, dass die individuellen Werthaltungen das Engagement beeinflusst haben und nicht umgekehrt. Einschneidende Erfahrungen könnten aber auch im späteren Erwachsenenalter zu Veränderungen in individuellen Werthaltungen führen (Hofer et al. 2010).

Zusammenfassend lässt sich sagen: Unterschiedliche persönliche Werthaltungen und nicht nur der individuelle Wert Solidarität stehen im Zusammenhang mit der Ausübung einer freiwilligen Tätigkeit. Diese Zusammenhänge unterscheiden sich zwischen gesellschaftlichen Gruppen. Anstrengungen, das Engagement bislang nicht oder weniger engagierter Gruppen zu erhöhen, sollten dies berücksichtigen. Auch hinsichtlich der Frage, ob Engagierte ihr Engagement weiterführen oder gar ausweiten oder abbrechen, können diese Zusammenhänge von Bedeutung sein. 


\section{Literatur}

Einolf, C. J. (2011). Gender Differences in the Correlates of Volunteering and Charitable Giving. Nonprofit and Voluntary Sector Quarterly, 40(6), 1092-1112.

Enquete-Kommission ,Zukunft des Bürgerschaftlichen Engagements' (2002). Bericht der Enquete-Kommission ,Zukunft des Bürgerschaftlichen Engagements!. Bürgerschaftliches Engagement: auf dem Weg in eine zukunftsfähige Bürgergesellschaft (Bundestagsdrucksache 14/8900). Berlin: Deutscher Bundestag.

Freund, A. M., Nikitin, J., \& Ritter, J. O. (2009). Psychological Consequences of Longevity. Human Development, 52(1), 1-37.

Grönlund, H., Holmes, K., Kang, C., Cnaan, R., Handy, F., Brudney, J., Haski-Leventhal, D., Hustinx, L., Kassam, M., Meijs, L. P. M., Pessi, A., Ranade, B., Smith, K., Yamauchi, N., \& Zrinščak, S. (2011). Cultural Values and Volunteering: A Cross-cultural Comparison of Students' Motivation to Volunteer in 13 Countries. Journal of Academic Ethics, 9(2), 87-106.

Hofer, M., Reinders, H., \& Fries, S. (2010). How values change. A goal-theoretical approach to explaining individual and societal value change. Zeitschrift für Entwicklungspsychologie und Pädagogische Psychologie, 42(1), 26-38.

Klages, H., \& Gensicke, T. (2006). Wertesynthese: Funktional oder Dysfunktional? Kölner Zeitschrift für Soziologie und Sozialpsychologie, 58(2), 332-351.

Klages, H., \& Gensicke, T. (2002). Wertewandel und bürgerschaftliches Engagement an der Schwelle zum 21. Jahrhundert. Speyer: Forschungsinstitut für öffentliche Verwaltung.

Reinders, H., \& Youniss, J. (2006). School-based required community service and civic development in adolescents. Applied Developmental Science, 10(1), 2-12.

Robinson, O. C. (2013). Values and adult age. Findings from two cohorts of the European Social Survey. European Journal of Ageing, 10(1), 11-23.

Rokeach, M., \& Ball-Rokeach, S. J. (1989). Stability and Change in American Value Priorities, 1968-1981. American Psychologist, 44(5), 775-784.

Sawyerr, O. O., Strauss, J., \& Yan, J. (2005). Individual value structure and diversity attitudes: The moderating effects of age, gender, race, and religiosity. Journal of Managerial Psychology, 20(6), 498-521.

Schwartz, S. H., \& Bilsky, W. (1990). Toward A Universal Psychological Structure of Human Values: Extensions and cross-cultural replications. Journal of Personality and Social Psychology, 58(5), 878-891.

Schwartz, S. H., \& Bilsky, W. (1987). Toward A Universal Psychological Structure of Human Values. Journal of Personality and Social Psychology, 53(3), 550-562.

Schwartz, S. H., \& Rubel, T. (2005). Sex differences in value priorities: Cross-cultural and multimethod studies. Journal of Personality and Social Psychology, 89(6), 1010-1028.

Welzel, C. (2009). Werte- und Wertewandelforschung. In: V. Kaina \& A. Römmele (Hrsg.) Politische Soziologie (S. 109-140). Wiesbaden: VS Verlag für Sozialwissenschaften.

Windsor, T. D., Anstey, K. J., \& Rodgers, B. (2008). Volunteering and Psychological Well-Being among Young-Old Adults: How Much Is Too Much? The Gerontologist, 48(1), 59-70.

Open Access Dieses Kapitel wird unter der Creative Commons Namensnennung 4.0 International Lizenz (http://creativecommons.org/licenses/by/4.0/deed.de) veröffentlicht, welche die Nutzung, Vervielfältigung, Bearbeitung, Verbreitung und Wiedergabe in jeglichem Medium und Format erlaubt, sofern Sie den/die ursprünglichen Autor(en) und die Quelle ordnungsgemäß nennen, einen Link zur Creative Commons Lizenz beifügen und angeben, ob Änderungen vorgenommen wurden.

Die in diesem Kapitel enthaltenen Bilder und sonstiges Drittmaterial unterliegen ebenfalls der genannten Creative Commons Lizenz, sofern sich aus der Abbildungslegende nichts anderes ergibt. Sofern das betreffende Material nicht unter der genannten Creative Commons Lizenz steht und die betreffende Handlung nicht nach gesetzlichen Vorschriften erlaubt ist, ist für die oben aufgeführten Weiterverwendungen des Materials die Einwilligung des jeweiligen Rechteinhabers einzuholen. 